TITLE:

\title{
Ab initio study of ferromagnetism in edged iron nanowires under axial strain
}

\section{$\operatorname{AUTHOR}(\mathrm{S})$ :}

Shimada, Takahiro; Ishii, Yoshiyuki; Kitamura, Takayuki

\section{CITATION:}

Shimada, Takahiro ...[et al]. Ab initio study of ferromagnetism in edged iron nanowires under axial strain. Physical Review B 2011, 84(17):

174405.

ISSUE DATE:

2011-11

URL:

http://hdl.handle.net/2433/151856

RIGHT:

(C)2011 American Physical Society. 
PHYSICAL REVIEW B 84, 174405 (2011)

\title{
$A b$ initio study of ferromagnetism in edged iron nanowires under axial strain
}

\author{
Takahiro Shimada, ${ }^{*}$ Yoshiyuki Ishii, and Takayuki Kitamura \\ Department of Mechanical Engineering and Science, Kyoto University, Sakyo-ku, Kyoto 606-8501, Japan
}

(Received 30 August 2011; revised manuscript received 20 October 2011; published 7 November 2011)

\begin{abstract}
We perform first-principles simulations of the magnetic and electronic properties of ferromagnetic (FM) body-centered cubic (bcc) iron nanowires with characteristic sharp edges consisting of (110) and (1 $\overline{1} 0)$ surfaces and their response to mechanical strain. An enhanced magnetic moment of $2.83 \mu_{B}$ is obtained at the edge of the FM nanowire. This enhancement originates from rearrangement of $d$ electrons from the minority-spin $t_{2 g}$ state to the majority-spin $t_{2 g}$ state due to a significant reduction in the number of nearest-neighbor atoms at the edge. The FM phase is the most energetically favorable phase in the nanowire even under relatively high axial strains, whereas the corresponding bulk material exhibits a FM-to-antiferromagnetic transition under the same loading conditions. During tension, a discontinuous change in the magnetic moment is observed at the surface and inside the nanowire due to a bcc-face-centered-cubic structural transition. In contrast, the magnetic moment at the edge is insensitive to applied strain. This is because the majority-spin state is fully occupied at the edge, which restricts the $t_{2 g}-e_{g}$ electron rearrangement to just the minority-spin state.
\end{abstract}

DOI: 10.1103/PhysRevB.84.174405

\section{INTRODUCTION}

In recent years, magnetic nanowires have attracted considerable attention as one-dimensional multifunctional materials. This is because they have distinctive magnetic properties that differ from those of the corresponding bulk materials ${ }^{1-3}$ and they are promising for technological applications (e.g., in ultrahigh-density magnetic storage devices, magnetic sensors, and nanomotors). ${ }^{4-7}$ Both the scientific interest in the unusual magnetic properties of nanoscale components and the increasing demand for miniaturization of magnetic devices are driving researchers to synthesize and investigate magnetic nanowires. Recent advances have enabled extremely small nanowires with diameters of only several nanometers to be fabricated. ${ }^{8-10}$ Magnetic iron nanowires fabricated along a surface step on a $\mathrm{W}(110)$ or Mo(110) substrate with a vicinal surface $^{11-15}$ characteristically possess a body-centered-cubic (bcc) crystal structure and atomically sharp edges consisting of (110) and (110) surfaces. ${ }^{15}$ Such sharp edges are commonly observed in other nanostructured bcc metals (e.g., corners of iron nanoislands ${ }^{16}$ and surface steps on tungsten and molybdenum $\left.{ }^{17}\right)$. Thus, (110)/(110) edges are characteristic nanostructures in bcc metals.

Since atomically sharp edges in magnetic iron nanowires have extremely low coordination numbers, the reduced interaction from neighboring atoms may cause different magnetic and electronic properties from those of the corresponding bulk material. In particular, because ultrathin nanowires have a high portion of edges and surfaces relative to the volume, structural effects are expected to dominate their magnetic properties. In addition, nanowires are often subjected to mechanical strain along their axes, which sometimes destabilizes the magnetic ordering. ${ }^{18-25}$ Thus, the magnetic properties of small iron nanowires with atomically sharp edges and the effect of axial strain on them are of fundamental interest in various fields.

Theoretical studies based on ab initio first-principles density functional theory calculations ${ }^{26,27}$ have provided comprehensive insight into magnetic nanostructures., ${ }^{2,3,28}$ Zelený et al. systematically investigated the structure and magnetism of nanowires and nanorods of transition metals. ${ }^{2,3} \mathrm{Jo}$ and Lee studied the magnetic and structural properties of ultrathin
PACS number(s): 75.75.-c, 73.22.-f, 31.15.A-

nanowires and atomic chains. ${ }^{28}$ However, to the best of our knowledge, the magnetism at $(110) /(1 \overline{1} 0)$ edges in bcc iron nanowires and the effect of strain on it has not been reported.

In this study, we perform $a b$ initio spin density functional theory calculations to investigate magnetism in bcc iron nanowires with atomically sharp edges consisting of (110) and (110) surfaces and the effect of axial strain on the magnetism. Section II describes the simulation procedure. Section III describes the energetic, magnetic, and electronic properties of iron nanowires with atomically sharp edges by focusing on the sharp edges. We also investigate the effect of wire size and axial strain on the magnetism of the nanowire. Finally, Sec. IV summarizes the results.

\section{COMPUTATIONAL DETAILS}

\section{A. Simulation method}

$A b$ initio first-principles calculations based on the spindensity-functional theory are performed using the Vienna $a b$ initio simulation package (VASP) code. ${ }^{29,30}$ The electronic wave functions are expanded in plane waves up to a cutoff kinetic energy of $300 \mathrm{eV}$. The electron-ion interaction is described by projector augmented wave (PAW) potentials ${ }^{31,32}$ that explicitly include the $\mathrm{Fe} 3 d$ and $4 s$ electrons in the valence states. Since the PAW approach realizes not only the high computational efficiency of the pseudopotential method but also the accuracy of all-electron methods (which do not suffer from problems associated with linearization of the core-valence exchange interaction), PAW potentials can accurately describe the magnetism at a transition metal surface. ${ }^{33}$ The generalized gradient approximation (GGA) proposed by Perdew, Burke, and Ernzerhof ${ }^{34}$ is employed to evaluate the exchange-correlation energy. The use of the GGA is essential to describe the structural and magnetic ground state of iron..$^{22,25,35}$

\section{B. Simulation models and procedure}

Figure 1 shows the simulation model of a bcc iron nanowire with atomically sharp edges consisting of (110) and (110) surfaces. The nanowire has a $3 \sqrt{2} a_{0} \times 3 \sqrt{2} a_{0}$ cross-sectional area, in which three $\sqrt{2} a_{0} \times \sqrt{2} a_{0} \times a_{0}$ bcc cells are arranged 

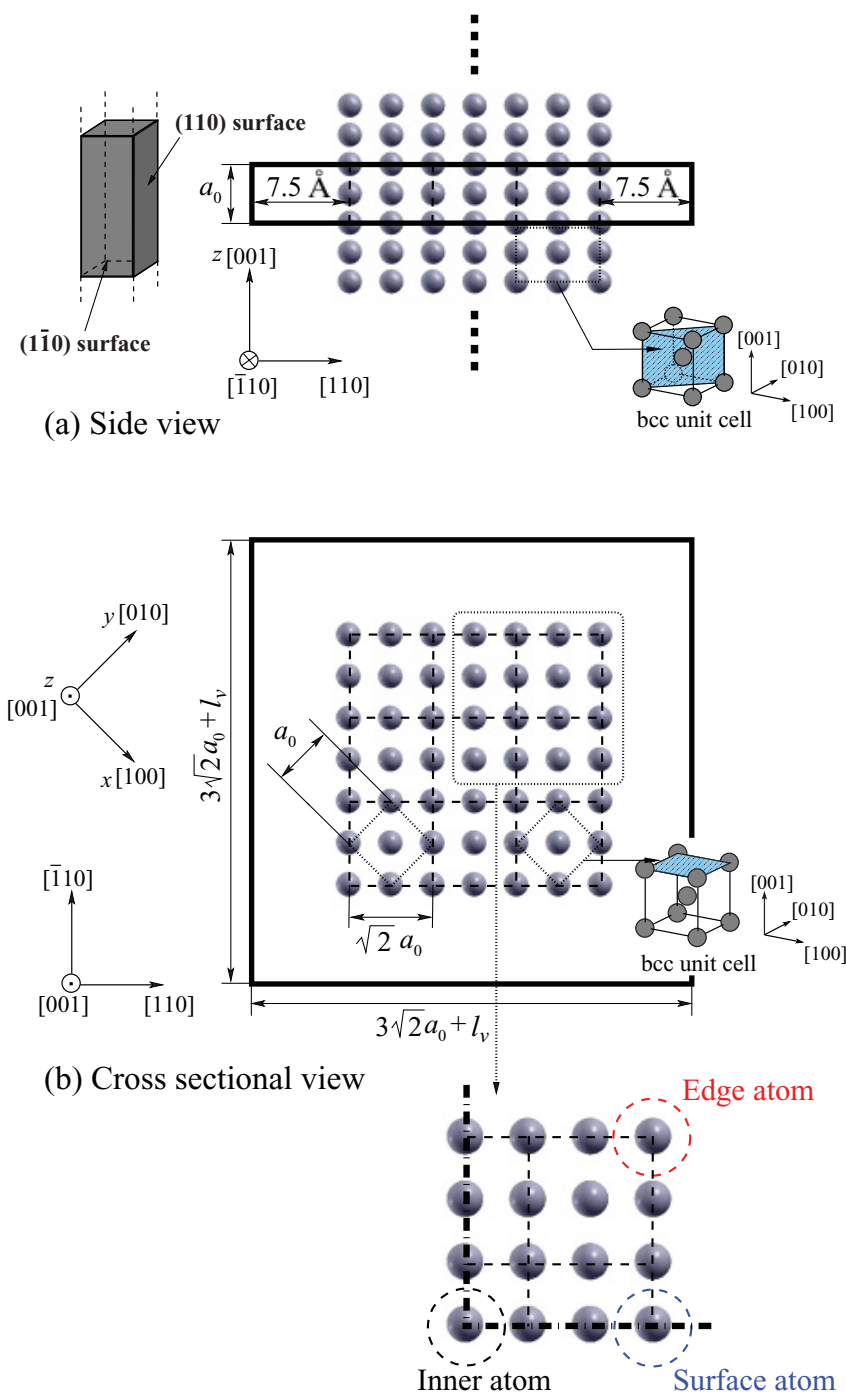

FIG. 1. (Color online) (a) Side and (b) cross-sectional views of simulation model of bcc iron nanowire with a $3 \times 3$ cell cross section. The nanowire is encased by (110) and (1 10$)$ surfaces. The solid black box represents the simulation cell. Atoms at the edge, on the surface, and inside the wire are referred to as edge, surface, and inner atoms, respectively.

in the [110] and [1 10] directions, respectively (see the dashed lines in Fig. 1). Here, $a_{0}$ is the theoretical lattice constant of bcc iron bulk, $a_{0}=2.835 \AA$. We refer to this model below as a $3 \times 3$ nanowire. Since three-dimensional periodic boundary conditions are applied, a vacuum region is introduced with a thickness of $l_{v}=15 \AA$ perpendicular to the wire axis to suppress undesirable interactions from neighboring nanowires. Thus, the simulation cell dimensions in the cross-sectional and axial directions are initially set to $3 \sqrt{2} a_{0}+l_{v}$ and $a_{0}$, respectively. Brillouin-zone integrations are performed with a $2 \times 2 \times 14$ Monkhorst-Pack $k$-point mesh. ${ }^{36}$ Atoms at the edge, on the surface, and inside the nanowire are referred to as "edge atoms," "surface atoms," and "inner atoms," respectively [see Fig. 1(b)]. To systematically investigate stable magnetic structures in the nanowire, we consider the possible magnetic orderings of the ferromagnetic (FM), nonmagnetic (NM), and antiferromagnetic (AFM) phases. The double-layer AFM $(\uparrow \uparrow \downarrow \downarrow \ldots)$ state $^{19,37}$ is more energetically favorable than the single-layer AFM $(\uparrow \downarrow \uparrow \downarrow$...) state in the nanowire. Thus, we refer to double-layered AFM ordering as the AFM state. The antiferromagnetic ordering is oriented parallel to the wire axis. In calculations of double-layer AFM nanowires, we use a simulation cell whose length is doubled in the axial $z$ direction (i.e., the $z$ cell dimension is $2 a_{0}$ ). We also investigate smaller nanowires with cross sections of $2 \times 2$ and $1 \times 1$ cells in the same manner to elucidate the finite-size effect.

To obtain the equilibrium structure, the atomic positions, and cell dimensions in the $z$ direction are fully relaxed using the conjugate gradient method and the quasi-Newton method until all the Hellmann-Feynman forces and the normal component of the stress $\sigma_{z z}$ are less than $2.5 \times 10^{-3} \mathrm{eV} / \AA$ and $1.0 \times$ $10^{-2} \mathrm{GPa}$, respectively.

To simulate the effect of tensile strain, a small incremental strain $\Delta \varepsilon_{z z}$ is applied stepwise in the axial $z$ direction to the simulation cell. At each strain, the atomic structure is fully relaxed under fixed cell dimensions.

\section{RESULTS AND DISCUSSION}

\section{A. Energetics and magnetism of iron nanowires with atomically sharp edges}

The FM phase was found to be the most stable phase for the $3 \times 3$ nanowire: The total energy difference per atom between the stable FM and the AFM phases $\Delta E_{\mathrm{AFM}-\mathrm{FM}}$ is $0.122 \mathrm{eV} /$ atom and that between the FM and the NM phases $\Delta E_{\mathrm{NM}-\mathrm{FM}}$ is $0.381 \mathrm{eV} /$ atom. The FM phase is also more energetically favorable than the AFM and NM phases for the smaller $2 \times 2$ and $1 \times 1$ nanowires. Hence, we focus on FM iron nanowires in the following discussion.

Here, we briefly discuss the formation energy of the edge structure. The edge energy $E_{\text {edge }}$ in the $3 \times 3$ nanowire is defined as the excess energy due to the formation of the edge. It can be calculated using

$$
E_{\text {edge }}=\frac{E_{\text {wire }}-E_{\text {bulk }} N-4 E_{\text {surf }} S}{4 a_{0}},
$$
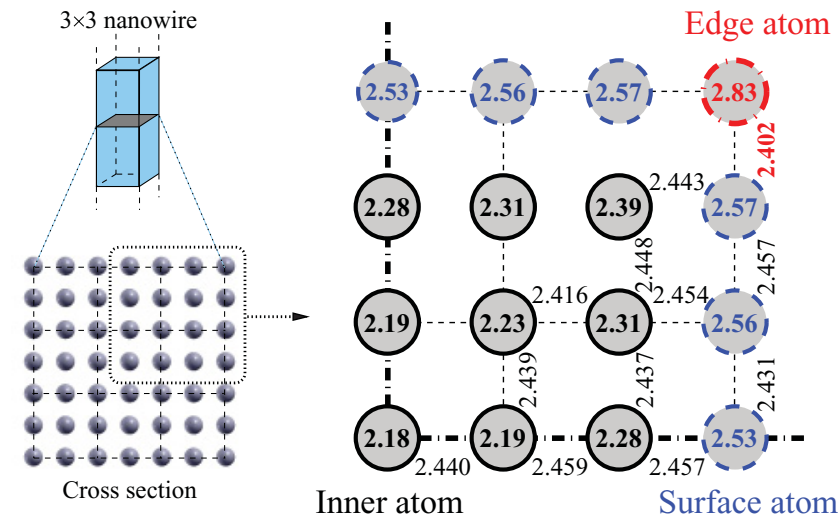

FIG. 2. (Color online) Distribution of local magnetic moments (in circles) and interatomic distances (between atoms) in $3 \times 3$ iron nanowire (in $\mu_{B}$ and $\AA$, respectively). Only the top right quarter of the cross section is shown due to symmetry. For comparison, the magnetic moments in iron bulk and at the (110) surface are $2.19 \mu_{B}$ and $2.52 \mu_{B}$ or $2.59 \mu_{B}$ (Refs. 39 and 40 ), respectively. 

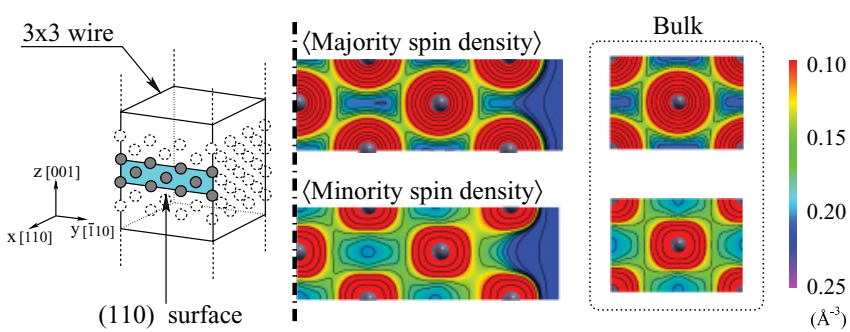

FIG. 3. (Color online) Majority-spin and minority-spin density distributions in the (110) surface plane of the $3 \times 3$ nanowire. For comparison, those of the bulk are also shown in the dashed square.

where $E_{\text {wire }}$ is the total energy of the $3 \times 3$ nanowire, $E_{\text {bulk }}$ is the total energy of the bcc iron bulk, $E_{\text {surf }}$ is the (110) surface energy, $N$ is the number of atoms in the simulation cell, and $S$ is the surface area. The calculated edge energy for the $\mathrm{Fe}(110) /(1 \overline{1} 0)$ edge is $3.85 \times 10^{-10} \mathrm{~J} / \mathrm{m}$. Face-centered-cubic metals have slightly higher edge energies than bcc iron. $\mathrm{Cu}$, $\mathrm{Au}, \mathrm{Co}$, and $\mathrm{Rh}(100) /(010)$ have edge energies of $E_{\text {edge }}=5.0$, $5.1,8.1$, and $9.5 \times 10^{-10} \mathrm{~J} / \mathrm{m}$, respectively. ${ }^{38}$

Figure 2 shows the distribution of local magnetic moments and interatomic distances in the $3 \times 3$ iron nanowire. The local magnetic moment is calculated by projecting the wave functions onto spherical harmonics within a sphere around each atom with a radius equal to that of the Wigner-Seitz sphere. The atom at the edge (indicated by the dotted-dashed red circle in Fig. 2) exhibits the highest magnetic moment of $2.83 \mu_{B}$ in the nanowire, which is about $30 \%$ larger than that of the bulk $\left(2.19 \mu_{B}\right)$, indicating that the magnetic moment is enhanced at the edge. This enhancement in the smaller $2 \times$ 2 and $1 \times 1$ nanowires is found to be $2.84 \mu_{B}$ and $2.85 \mu_{B}$, respectively. In addition, the edge atom possesses the shortest interatomic distance of $2.402 \AA$, while others range from 2.43 to $2.46 \AA$. The local magnetic moment at the surface (indicated by the dashed blue circles in Fig. 2) is relatively smaller [(2.53$\left.2.57) \mu_{B}\right]$, being nearly equal to that $\left(2.52 \mu_{B}\right.$ or $\left.2.59 \mu_{B}\right)$ at the corresponding (110) surface. ${ }^{39,40}$

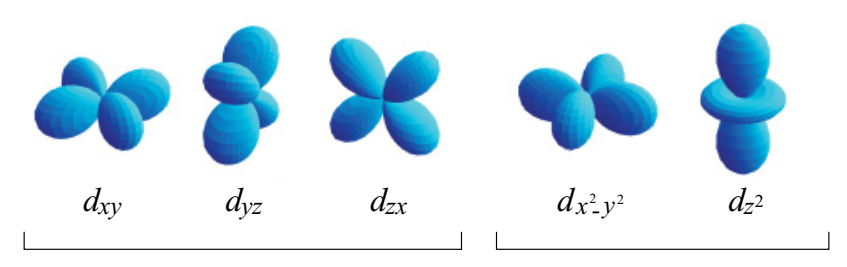

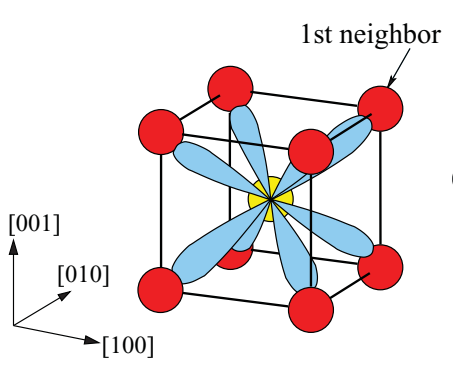

(a) Hybridized $t_{2 g}$ orbit

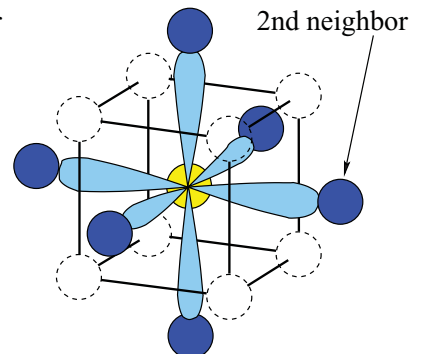

(b) $e_{g}$ orbit
FIG. 4. (Color online) Schematic illustrations of (a) hybridized $t_{2 g}\left(d_{x y}, d_{y z}\right.$, and $\left.d_{z x}\right)$ and (b) $e_{g}\left(d_{x^{2}-y^{2}}\right.$ and $\left.d_{z^{2}}\right)$ orbitals in bcc iron.
The magnetic moment inside the nanowire $\left(2.18 \mu_{B}\right.$ for the inner atom) is almost the same as that $\left(2.19 \mu_{B}\right)$ of bulk iron. Thus, the effective range of the edge is estimated to be about 4 Å.

Figure 3 shows the majority-spin and minority-spin density distributions in the (110) plane of the $3 \times 3$ nanowire; it also shows those of the bulk for comparison. There is a distinct difference between the majority-spin and minorityspin density distributions: the majority spin is distributed almost spherically and isotropically in both the nanowire and the bulk, whereas the minority spin has an anisotropic distribution in which the electrons are concentrated near the nearest neighbors. At the edge of the nanowire, the minority
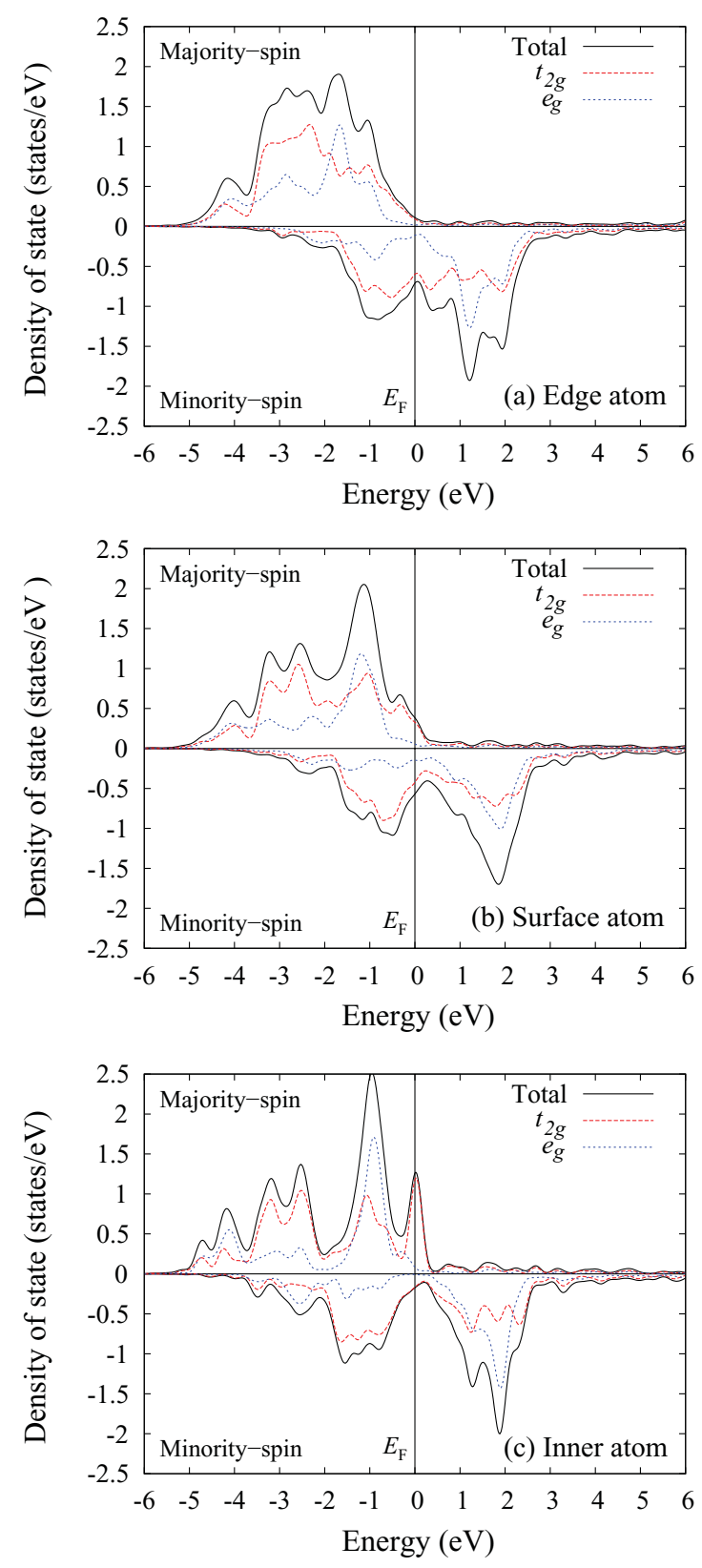

FIG. 5. (Color online) Total $d$ and $t_{2 g}-e_{g}$ decomposed electronic local densities of states of the (a) edge, (b) surface, and (c) inner atoms in the $3 \times 3$ nanowire. The vertical solid line indicates the Fermi level $E_{F}$. 


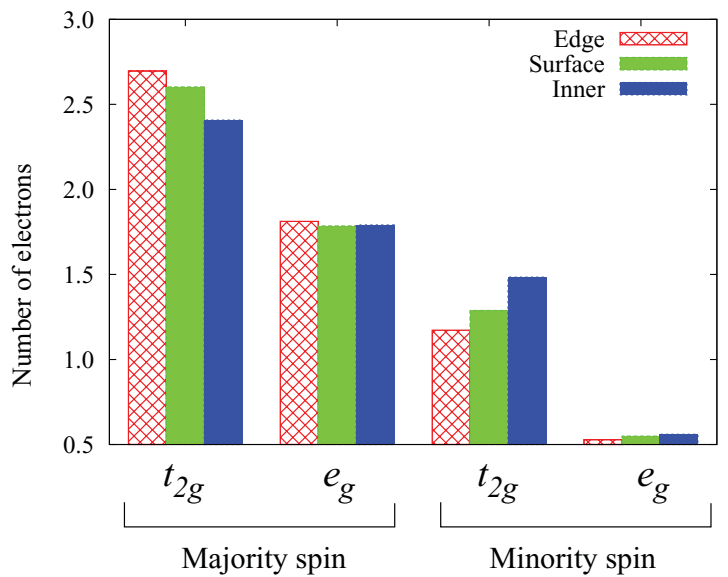

FIG. 6. (Color online) Number of majority-spin and minorityspin electrons for the $t_{2 g}$ and $e_{g}$ states at the edge, surface, and inner atoms.

spin loses directionality toward the vacuum region because of the absence of nearest neighbors. Considering the electronic state of $d$ orbitals in the bcc structure, which dominates the magnetic and structural properties of iron, the five $d$ orbitals $d_{x y}, d_{y z}, d_{z x}, d_{x^{2}-y^{2}}$, and $d_{z^{2}}$ reduce in the bcc crystal to the hybridized $t_{2 g}\left(d_{x y}, d_{y z}\right.$, and $\left.d_{z x}\right)$ and $e_{g}\left(d_{x^{2}-y^{2}}\right.$ and $\left.d_{z^{2}}\right)$ states according to crystal field theory, ${ }^{41,42}$ as schematically illustrated in Fig. 4. The $t_{2 g}$ electrons are distributed toward the eight nearest neighbors (red spheres in Fig. 4) in the bcc lattice to form $d d \sigma$ bonds, ${ }^{43}$ whereas the $e_{g}$ electrons are directed toward the second-nearest neighbors (blue spheres in Fig. 4). Considering these characteristics of the $t_{2 g}$ and $e_{g}$ electron distributions, the $t_{2 g}$ state should predominantly contribute to the minority-spin density distribution along the nearest-neighbor direction.

Figure 5 shows the spin-polarized local electronic density of states (DOS) of the total $d$ and $t_{2 g}-e_{g}$ decomposed states of the edge, surface, and inner atoms in the $3 \times 3$ nanowire. The local DOS is calculated by projecting the wave functions onto the spherical harmonics in the spheres around each atom. For the majority spin of all the atoms, both the $t_{2 g}$ and $e_{g}$ states are highly occupied. This indicates that both the $t_{2 g}$ and $e_{g}$ states are almost equally occupied, which results in the isotropic distribution of the majority-spin density in Fig. 3. For the minority spin, on the other hand, the $t_{2 g}$ state is highly occupied, whereas the $e_{g}$ state is mainly distributed above the Fermi level. This corresponds to the directional minority-spin density distribution toward the nearest neighbors. For the minority spin, the DOS of the $t_{2 g}$ states of the inner atom distributed above and below $E_{F}$ exhibits a broader bandwidth (i.e., the range of energy levels that a state occupies). On the other hand, the $t_{2 g}$ DOS of the edge atom is localized around $E_{F}$, resulting in a reduced bandwidth. It is well known that the bandwidth decreases as the coordination number decreases. Because the edge atom has fewer nearest neighbors than
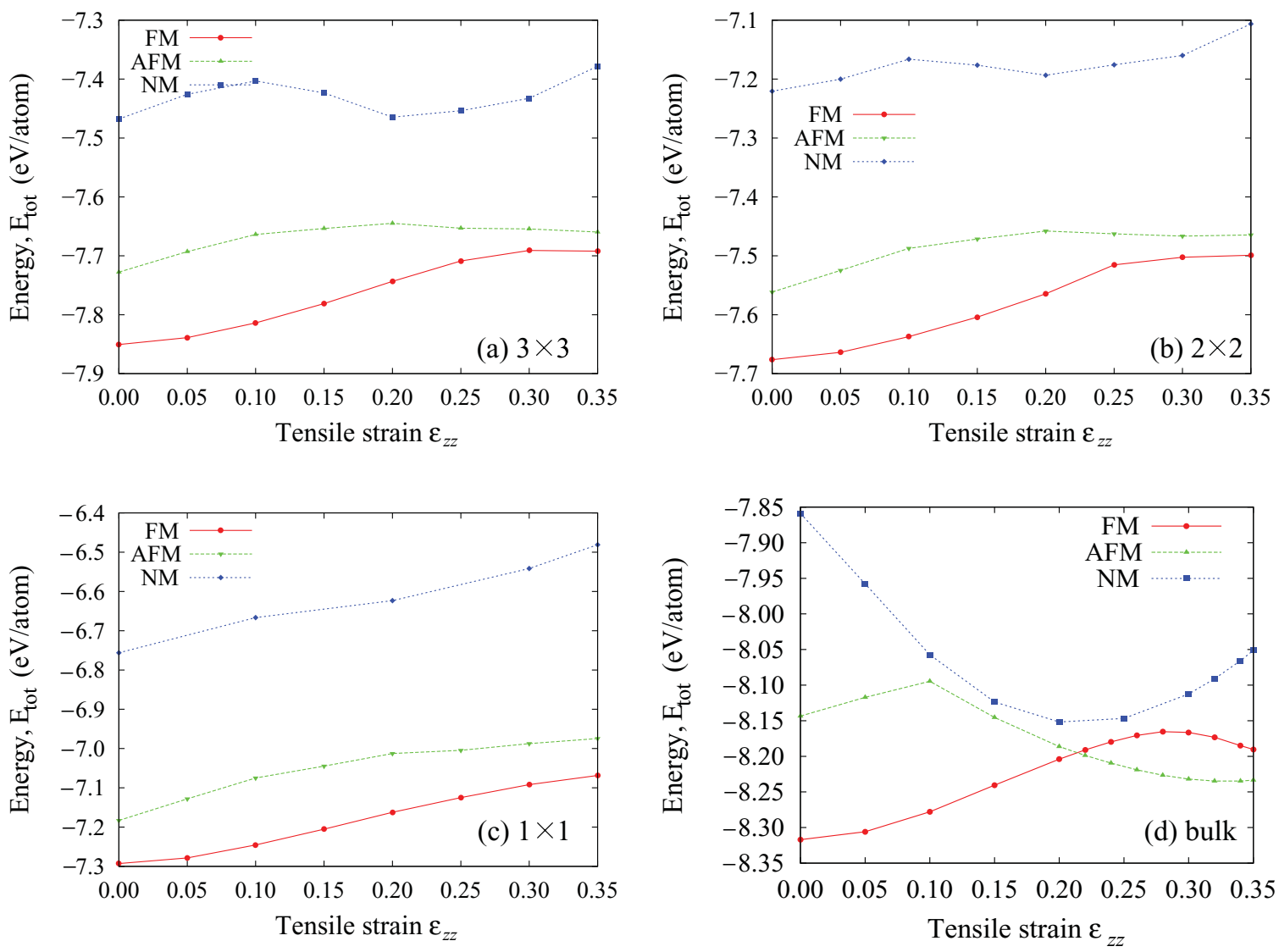

FIG. 7. (Color online) Total energy per atom for (a) $3 \times 3$, (b) $2 \times 2$, and (c) $1 \times 1$ nanowires in the FM, AFM, and NM phases as a function of axial tensile strain $\varepsilon_{z z}$ along [001]. For comparison, the same relationship in bulk Fe under [001] tension is shown in (d). 
inner atoms (four as opposed to eight), the bandwidth of the corresponding $t_{2 g}$ states decreases. A similar reduction in bandwidth is also found for surface atoms, but it is less than that of the edge atom because surface atoms lose fewer nearest neighbors (six as opposed to eight).

Figure 6 shows the number of majority-spin and minorityspin electrons for the $t_{2 g}$ and $e_{g}$ states at the edge, surface, and inner atoms. Comparison of the edge atom with an inner atom reveals a remarkable increase (reduction) in the number of electrons in the majority-spin (minority-spin) $t_{2 g}$ state. This indicates that the electrons of the edge atom change from minority-spin $t_{2 g}$ to majority-spin $t_{2 g}$ states. This enhances the magnetic moment at the edge atom because the magnetic moment is defined as the difference between the number of majority-spin and minority-spin electrons.

\section{B. Influence of axial tensile strain}

Figures $7(\mathrm{a})-7$ (c) show the total energy per atom in the FM, AFM, and NM phases of the $3 \times 3,2 \times 2$, and $1 \times 1$ nanowires, respectively, as a function of the axial tensile strain $\varepsilon_{z z}$. For comparison, Fig. 7(d) shows the relationship in bulk iron under [001] tension (i.e., the same loading as that applied to the nanowires). For bulk iron, the magnetic ground state is FM under strain-free conditions, but it becomes AFM when $\varepsilon_{z z}=0.22$ because the [001] tension causes the crystal lattice to transform from bcc to fcc, which is known as the Bain transformation path. ${ }^{19,25,44}$ On the other hand, the FM state in the nanowires is always more energetically favorable than the AFM state within the present range of applied strains. Thus, the nanowires do not exhibit the FM-AFM magnetic phase transition observed in the bulk.

Figure 8(a) shows the tetragonality of the lattice $c / a$ around the edge, surface, and inner atoms in the FM $3 \times 3$ nanowire as a function of axial tensile strain $\varepsilon_{z z}$, where $a$ and $c$ denote the lattice spacings in the lateral $x$ and $y$ directions and in the $z$ direction, respectively [see the schematic illustration in Fig. 8(a)]. The tetragonality is almost $c / a=1$ when no strain is applied, indicating that the nanowire is initially has a bcc structure. As an axial strain is applied, the tetragonality increases. The edge atom exhibits a larger tetragonality than the surface and inner atoms. An abrupt increase in the tetragonality is observed in the range $\varepsilon_{z z}=0.30-0.31$ in which $c / a$ is slightly larger than $\sqrt{2}$, except for the edge atom. Since the tetragonality $c / a=\sqrt{2}$ corresponds to the fcc configuration [see the lattice in Fig. 8(a)], this abrupt increase is related to the structural transformation of the nanowire from the body-centered tetragonal (bct) to the fcc phase. Such an abrupt change in tetragonality has also been observed when bulk bcc iron transforms into the fcc structure along the Bain path. ${ }^{19,25}$

Figure 8(b) shows the magnitude of the magnetic moments of the edge, surface, and inner atoms in the FM $3 \times 3$ nanowire as a function of the axial tensile strain $\varepsilon_{z z}$. The applied tensile strain tends to enhance the magnetic moments of both the inner and surface atoms, although surface atoms exhibit a more moderate increase with strain than that of inner atoms. The magnetic moments decrease dramatically between $\varepsilon_{z z}=0.30$ and 0.31 , where the structural change from bct to fcc occurs. This suggests that the sharp reduction in the magnetic moment is due to the structural transition. Previous
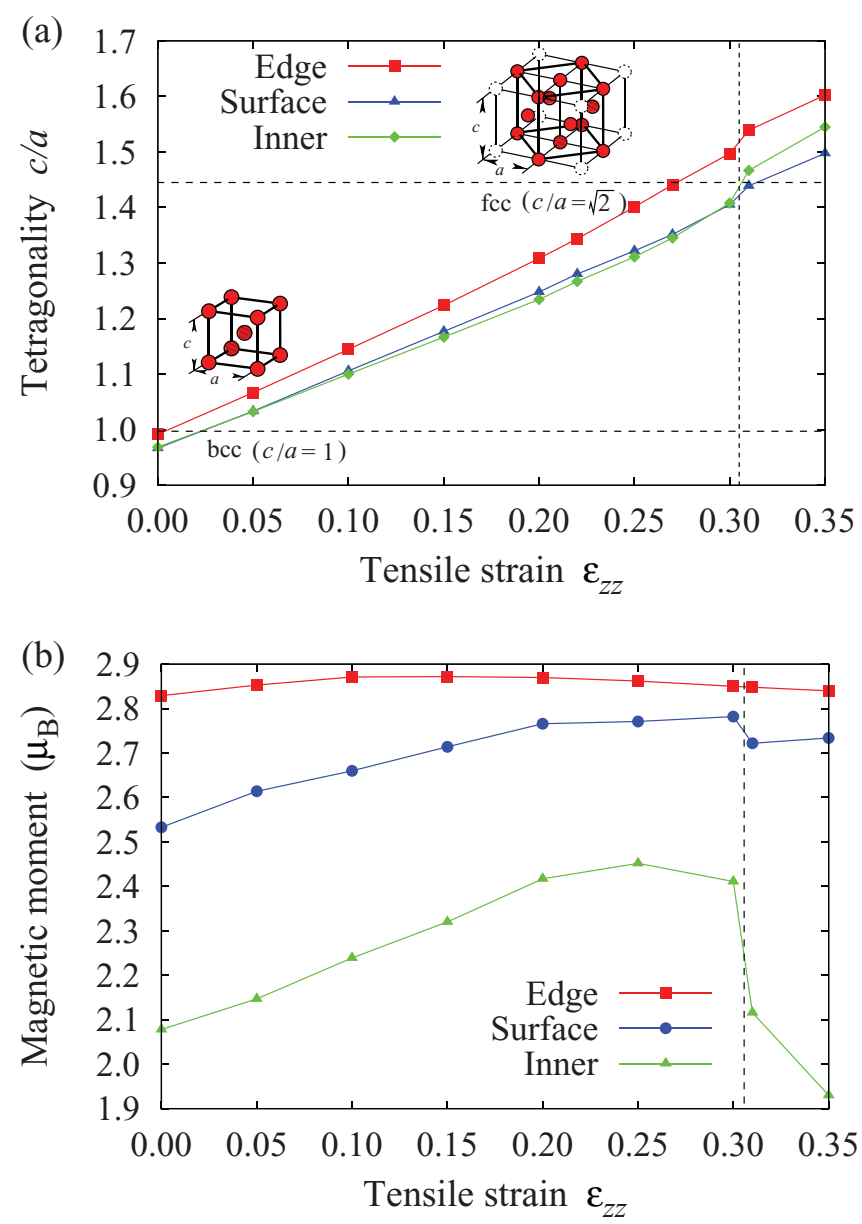

FIG. 8. (Color online) (a) Tetragonality of lattice $c / a$ around the edge, surface, and inner atoms in the FM $3 \times 3$ nanowire as a function of axial tensile strain $\varepsilon_{z z} . a$ and $c$ denote the lattice spacings in the lateral $x$ and $y$ directions and in the axial $z$ direction, respectively. (b) Magnitude of magnetic moment of the edge, surface, and inner atoms in the FM $3 \times 3$ nanowire as a function of axial tensile strain $\varepsilon_{z z}$.

studies have reported a similar reduction in magnetic moment in bulk iron during the Bain bcc-fcc transformation. ${ }^{19,25}$ In contrast, the magnetic moment of the edge atom is almost unchanged by the applied strain. Even at the bct-fcc transition strain, the magnetic moment of the edge atom remains constant despite the abrupt change in the crystal structure.

Figure 9 shows the change in the number of majority-spin and minority-spin electrons in the $t_{2 g}$ and $e_{g}$ states as a function of the axial tensile strain $\varepsilon_{z z}$ of the inner and edge atoms. For the inner atom, the number of minority-spin and majority-spin electrons changes discontinuously near the transition strain of $\varepsilon_{z z}=0.30-0.31$, which leads to an abrupt change in the magnetic moment of the inner atom. This clearly results from the structural change from bct to fcc because the electronic $t_{2 g}$ and $e_{g}$ states are characterized by the crystalline structure, ${ }^{41,42}$ as shown in Fig. 4. On the other hand, for the edge atom, there is no discontinuous change in the magnetic moment despite the bct-fcc transition. In addition, the number of majority-spin electrons does not change when strain is applied. Thus, the electron rearrangement is limited to within the minorityspin state: the electrons are exchanged only between the 


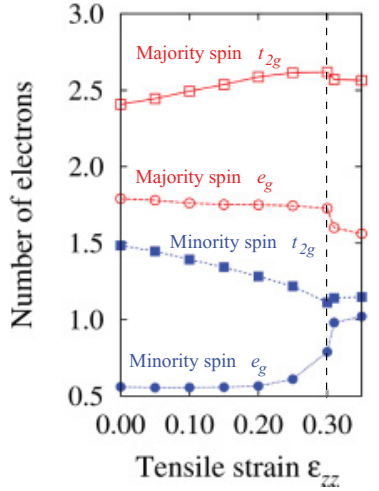

(a) Inner atom

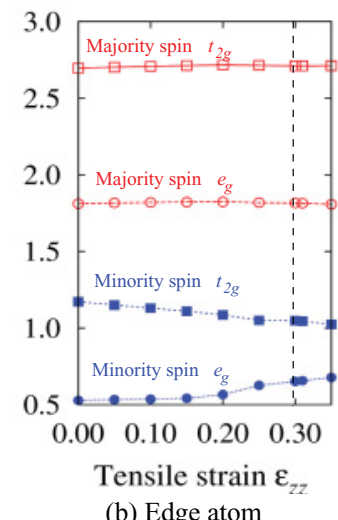

(b) Edge atom

FIG. 9. (Color online) Change in the number of majority-spin (red) and minority-spin (blue) electrons in the $t_{2 g}$ and $e_{g}$ states as a function of axial tensile strain $\varepsilon_{z z}$ of (a) inner and (b) edge atoms. The vertical dashed lines indicate the point at which the bct-fcc transition occurs.

minority-spin $t_{2 g}$ and $e_{g}$ states. Figure 10 plots the change in the local electronic density of states of the $t_{2 g}$ and $e_{g}$ states between axial strains of $\varepsilon_{z z}=0.30$ and 0.31 for the inner and edge atoms. Unoccupied $t_{2 g}$ and $e_{g}$ states exist for both the majority

and minority spins in the inner atom during the structural transition, whereas the majority-spin state remains fully occupied in the edge atom because of its lower coordination number. Thus, the electron rearrangement of the edge atom is limited to within the minority-spin state, which accounts for the insensitivity of the magnetic moment to the applied strain.

\section{CONCLUSION}

$A b$ initio first-principles spin density functional theory calculations within the GGA were performed to investigate the magnetism of nanowires with atomically sharp edges and having (110) and (110) surfaces and its response to the axial strain. At the edge of the strain-free FM nanowire, the magnetic moment is enhanced $\left(2.83 \mu_{B}\right)$ relative to that $\left(2.19 \mu_{B}\right)$ of the bulk. This magnetic enhancement originates from the rearrangement of the $d$ electrons from the minority-spin $t_{2 g}$ to the majority-spin $t_{2 g}$ state at the edge due to the low coordination number for nearest neighbors. The FM phase is always more energetically favorable in the nanowire even under relatively high axial strain, although the corresponding bulk material exhibits a FM-to-AFM transition under the same loading conditions. In simulations of the effect of applying a strain, a discontinuous change in the magnetic moments

(a) Inner atom
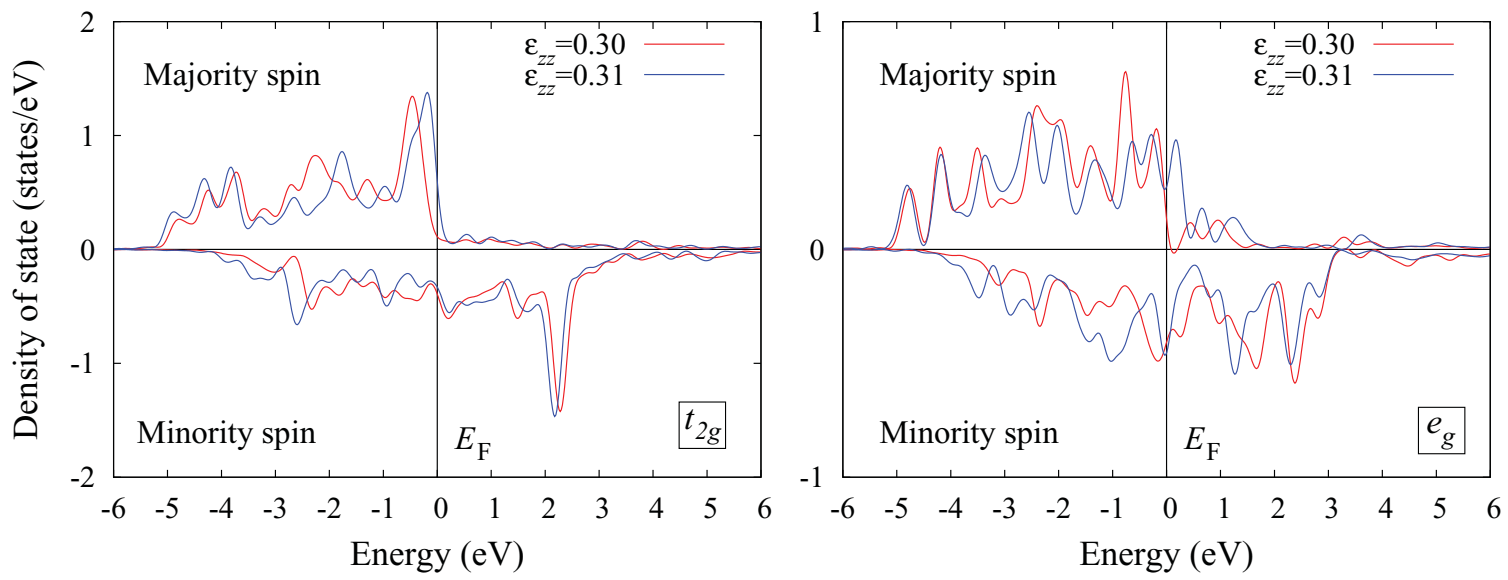

(b) Edge atom
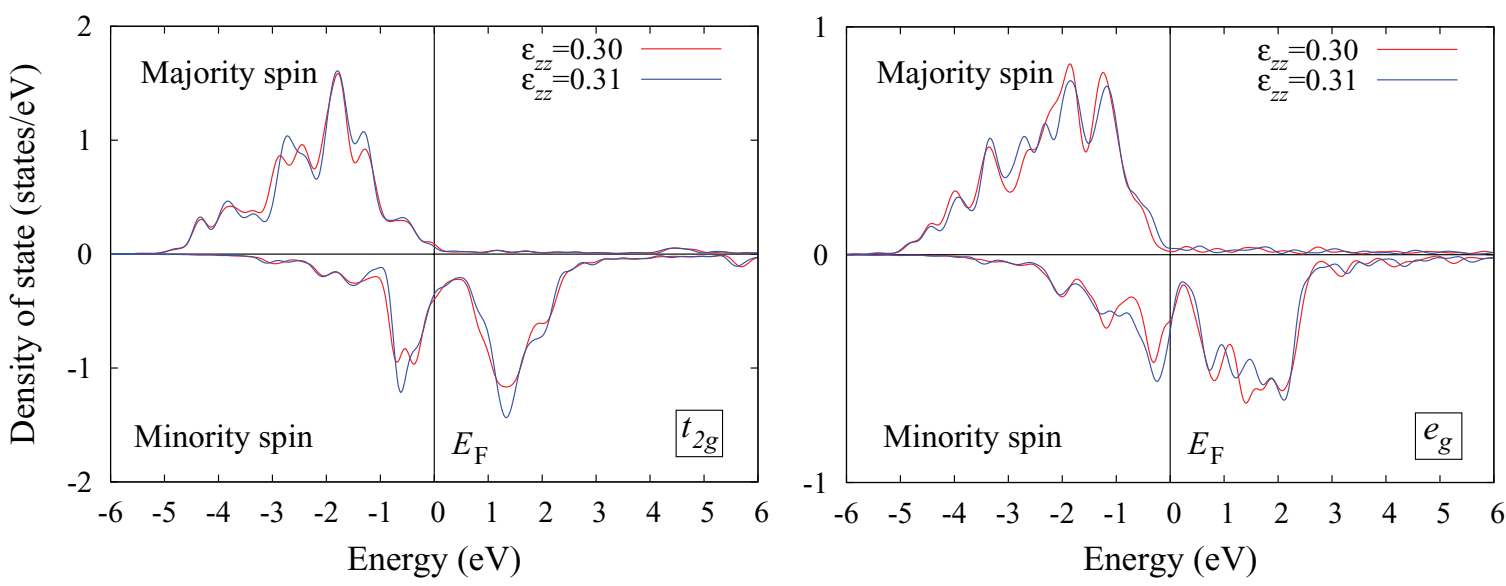

FIG. 10. (Color online) Change in local electronic density of states of the $t_{2 g}$ and $e_{g}$ states before $\left(\varepsilon_{z z}=0.30\right)$ and after $\left(\varepsilon_{z z}=0.31\right)$ the bct-fcc structural transition for (a) inner and (b) edge atoms. 
was observed at the surface and inside the nanowire due to a bcc-fcc structural transition, whereas the magnetic moment at the edge is almost unchanged by the strain. This insensitivity at the edge to strain arises from the $t_{2 g}-e_{g}$ electron exchange being restricted to only the minority-spin state due to the majority-spin state being fully occupied.

\section{ACKNOWLEDGMENTS}

This work was supported in part by a Grant-in-Aid for Scientific Research (S) (Grant No. 21226005) and a Grant-inAid for Young Scientists (A) (Grant No. 23686023) from the Japan Society for the Promotion of Science (JSPS). *shimada@cyber.kues.kyoto-u.ac.jp

${ }^{1}$ G. Autès, C. Barreteau, D. Spanjaard, and M. C. Desjonquères, J. Phys.: Condens. Matter 18, 6785 (2006).

${ }^{2}$ M. Zelený, M. Šob, and J. Hafner, Phys. Rev. B 79, 134421 (2009).

${ }^{3}$ M. Zelený, M. Šob, and J. Hafner, Phys. Rev. B 80, 144414 (2009).

${ }^{4}$ G. A. Prinz, Science 282, 1660 (1998).

${ }^{5}$ F. Li, R. M. Metzger, and W. D. Doyle, IEEE Trans. Magn. 33, 3715 (1997).

${ }^{6}$ K. K. Lew and J. M. Redwing, J. Cryst. Growth 254, 14 (2003).

${ }^{7}$ Y. C. Wang, I. C. Leu, and M. H. Hon, J. Cryst. Growth 237, 564 (2002).

${ }^{8}$ S. G. Yang, H. Zhu, D. L. Yu, Z. Q. Jin, S. L. Tang, and Y. W. Du, J. Magn. Magn. Mater. 222, 97 (2000).

${ }^{9}$ X. Y. Zhang, G. H. Wen, Y. F. Chan, R. K. Zheng, X. X. Zhang, and N. Wang, Appl. Phys. Lett. 83, 3341 (2003).

${ }^{10}$ D. J. Sellmyer, M. Zheng, and R. Skomski, J. Phys.: Condens. Matter 13, R433 (2001).

${ }^{11}$ B. Borca, O. Fruchart, E. Kritsikis, F. Cheynis, A. Rousseau, P. David, C. Meyer, and J. C. Toussaint, J. Magn. Magn. Mater. 322, 257 (2010).

${ }^{12}$ J. Hauschild, U. Gradmann, and H. J. Elmers, Appl. Phys. Lett. 72, 3211 (1998).

${ }^{13}$ A. Dallmeyer, C. Carbone, W. Eberhardt, C. Pampuch, O. Rader, W. Gudat, P. Gambardella, and K. Kern, Phys. Rev. B 61, R5133 (2000).

${ }^{14}$ O. Fruchart, M. Eleoui, J. Vogel, P. O. Jubert, A. Locatelli, and A. Ballestrazzi, Appl. Phys. Lett. 84, 1335 (2004).

${ }^{15}$ B. Borca, O. Fruchart, F. Cheynis, M. Hasegawa, and C. Meyer, Surf. Sci. 601, 4358 (2007).

${ }^{16}$ O. Fruchart, P. O. Jubert, M. Eleoui, F. Cheynis, B. Borca, P. David, V. Satonacci, A. Liénard, M. Hasegawa, and C. Meyer, J. Phys.: Condens. Matter 19, 053001 (2007).

${ }^{17}$ J. P. Piece, E. W. Plummer, and J. Shen, Appl. Phys. Lett. 81, 1890 (2002).

${ }^{18}$ H. C. Herper, E. Hoffmann, and P. Entel, Phys. Rev. B 60, 3839 (1999).
${ }^{19}$ M. Friák, M. Šob, and V. Vitek, Phys. Rev. B 63, 052405 (2001).

${ }^{20}$ L. Tsetseris, Phys. Rev. B 72, 012411 (2005).

${ }^{21}$ M. Friák and M. Šob, Phys. Rev. B 77, 174117 (2008).

${ }^{22}$ M. Zelený, D. Legut, and M. Šob, Phys. Rev. B 78, 224105 (2008).

${ }^{23}$ S. V. Okatov, A. R. Kuznetsov, Y. N. Gornostyrev, V. N. Urtsev, and M. I. Katsnelson, Phys. Rev. B 79, 094111 (2009).

${ }^{24}$ A. Hashibon, P. Schravendijk, C. Elsässer, and P. Gumbsch, Philos. Mag. 89, 3413 (2009).

${ }^{25}$ T. Shimada, Y. Ishii, and T. Kitamura, Phys. Rev. B 81, 134420 (2010).

${ }^{26}$ P. Hohenberg and W. Kohn, Phys. Rev. 136, B864 (1964).

${ }^{27}$ W. Kohn and L. Sham, Phys. Rev. 140, A1133 (1965).

${ }^{28}$ C. Jo and J. I. Lee, Phys. Status Solidi B 241, 1427 (2004).

${ }^{29}$ G. Kresse and J. Hafner, Phys. Rev. B 47, 558 (1993).

${ }^{30}$ G. Kresse and J. Furthmüller, Phys. Rev. B 54, 11169 (1996).

${ }^{31}$ P. E. Blöchl, Phys. Rev. B 50, 17953 (1994).

${ }^{32}$ G. Kresse and D. Joubert, Phys. Rev. B 59, 1758 (1999).

${ }^{33}$ G. Kresse, W. Bergermayer, and R. Podloucky, Phys. Rev. B 66, 146401 (2002).

${ }^{34}$ J. P. Perdew, K. Burke, and M. Ernzerhof, Phys. Rev. Lett. 77, 3865 (1996).

${ }^{35}$ E. G. Moroni, G. Kresse, J. Hafner, and J. Furthmüller, Phys. Rev. B 56, 15629 (1997).

${ }^{36}$ H. J. Monkhorst and J. D. Pack, Phys. Rev. B 13, 5188 (1976).

${ }^{37}$ D. Spišák and J. Hafner, Phys. Rev. B 61, 16129 (2000).

${ }^{38}$ J. C. W. Swart, P. van Helden, and E. van Steen, J. Phys. Chem. C 111, 4998 (2007).

${ }^{39}$ P. Błoński and A. Kiejna, Surf. Sci. 601, 123 (2007).

${ }^{40}$ M. Spencer, A. Hung, I. Snook, and I. Yarovsky, Surf. Sci. 540, 420 (2003).

${ }^{41}$ J. B. Goodenough, Phys. Rev. 120, 67 (1960).

${ }^{42}$ Y. Q. Xie, Acta Metall. Mater. 42, 3705 (1994).

${ }^{43}$ T. E. Jones, M. E. Eberhart, and D. P. Clougherty, Phys. Rev. Lett. 100, 017208 (2008).

${ }^{44}$ M. Marsman and J. Hafner, Phys. Rev. B 66, 224409 (2002). 